\section{Fontes de obtenção de medicamentos para hipertensão e diabetes no Brasil: resultados de inquérito telefônico nas capitais brasileiras e no Distrito Federal, 2011}

\author{
Sources of medicines for hypertension and \\ diabetes in Brazil: telephone survey results \\ from Brazilian state capitals and the \\ Federal District, 2011
}

Fuentes de obtención de medicamentos para la hipertensión y diabetes en Brasil: resultados de una encuesta telefónica en las capitales brasileñas y el Distrito Federal, 2011
Karen Sarmento Costa 1,2

Priscila Maria S. Bergamo Francisco 1 Débora Carvalho Malta ${ }^{3}$ Marilisa Berti de Azevedo Barros 1
${ }^{1}$ Faculdade de Ciências Médicas, Universidade Estadual de Campinas, Campinas, Brasil.

2 Secretaria de Ciência Tecnologia e Insumos Estratégicos, Ministério da Saúde, Brasília, Brasil. 3 Secretaria de Vigilância em Saúde, Ministério da Saúde, Brasília, Brasil.

\author{
Correspondência \\ K. S. Costa \\ Rua Nélson César de Oliveira \\ 170, São José dos Campos, SP \\ 12240-220, Brasil. \\ karen.costa@gmail.com
} em tratamento medicamentoso; 45,8\% dos hipertensos obtiveram medicamento nas unidades de saúde públicas, 15,9\% no Farmácia Popular 38,3\% em drogarias/farmácias e outras fontes. Entre os diabéticos, encontrou-se 54,4\%; 16,2\%; e 29,4\%, respectivamente. Nas unidades de saúde os percentuais foram mais elevados entre os menos escolarizados, cor de pele preta ou parda e sem plano privado de saúde, e as prevalências de obtenção na Farmácia Popular, drogarias/farmácias e outras fontes foram mais elevadas entre os mais escolarizados, cor de pele branca e com plano privado. O acesso às diferentes fontes de medicamentos apresentou disparidades entre as regiões e capitais brasileiras e entre os segmentos sociais da população.

Uso de Medicamentos; Acesso aos Serviços de Saúde; Hipertensão; Diabetes Mellitus 


\section{Introdução}

Estimativas da Organização Mundial da Saúde (OMS) apontam que as doenças crônicas não transmissíveis (DCNT), consideradas como um dos mais desafiadores problemas da saúde pública global, foram responsáveis por $63 \%$ dos 36 milhões de mortes ocorridas no mundo em 2008 1. Segundo Goulart 2, duas em cada três mortes ao ano, em todo o mundo, podem ser atribuídas às DCNT, sendo que quatro quintos destes óbitos (80\%) ocorrem em países de baixa e média rendas, e um terço atinge pessoas em idade produtiva, com menos de 60 anos. No Brasil, as DCNT são também a principal causa de mortalidade e afetam de forma mais intensa indivíduos pertencentes a grupos vulneráveis, com baixa escolaridade e menor renda 3,4 .

Dados da Pesquisa Nacional por Amostra de Domicílios (PNAD 2008) revelaram crescimento de $37 \%$ na prevalência do diabetes e de $15 \%$ na de hipertensão no país, no período de 2003 a 2008 4, aumento que impulsiona o consumo de medicamentos anti-hipertensivos e antidiabéticos. O tratamento medicamentoso, quando adequadamente conduzido, possibilita o controle das DCNT, redução da morbimortalidade e melhoria da qualidade de vida dos usuários portadores dessas condições 5 .

O Estado brasileiro estabeleceu o Plano de Ações Estratégicas para o Enfrentamento das Doenças Crônicas Não Transmissíveis, para o período 2011-2022, que contém definições e priorizações de ações e investimentos para detecção e controle dessas doenças e de seus fatores de risco 6 .

Diferentes estratégias têm sido implementadas pelo Estado brasileiro buscando viabilizar as diretrizes das políticas farmacêuticas no país 7,8 como: ampliação do financiamento público para maior disponibilidade de medicamentos nos serviços de saúde do Sistema Único de Saúde (SUS) 9, promulgação de instrumentos regulatórios relativos ao acesso aos medicamentos, estruturação da assistência farmacêutica e desenvolvimento de um programa nacional para qualificar a assistência farmacêutica no SUS 9, entre outras medidas.

Dentre essas iniciativas foi criado o Programa Farmácia Popular do Brasil (PFPB) em 2004 10, visando a ampliar ações de universalização do acesso da população aos medicamentos, por meio de atendimento oferecido a pessoas usuárias ou não de serviços públicos de saúde. Desde a sua institucionalização, o programa incorporou modificações sucessivas 11. Em 2011, passou a garantir a gratuidade de medicamentos para o tratamento da hipertensão, diabetes e asma em todas as farmácias do país credenciadas ao programa e nas suas unidades próprias, como parte de um programa conhecido como Saúde Não tem Preço.

Nesse contexto, reconhece-se que o acesso aos medicamentos envolve uma complexa rede de atores públicos e privados, no contexto econômico, político e social dos países nos quais são formulados modelos e sistemas de saúde para efetivar tal acesso, envolvendo várias combinações de provisão de serviços e financiamentos estatal e privado 12,13. Dentre as dimensões específicas do acesso - disponibilidade física do produto, acessibilidade, acessibilidade geográfica, aceitabilidade ou satisfação 13 - a disponibilidade física do produto é básica e precisa compatibilizar tipo/quantidade de produtos e serviços existentes com tipo/quantidade de produtos e serviços necessários. No presente estudo, foi selecionado para análise um aspecto da disponibilidade física, relativo às fontes de obtenção dos medicamentos para tratamento da hipertensão e diabetes, nas capitais brasileiras e no Distrito Federal.

Os indicadores de acesso aos medicamentos para o tratamento de DCNT consideradas prioritárias permitem aferir em que medida os serviços públicos e privados de saúde, de diferentes países, estão sendo capazes de prover assistência farmacêutica aos pacientes com estas doenças 14. Por essa razão, em 2011, questões relacionadas ao uso dos medicamentos para hipertensão e diabetes, e às fontes de obtenção destes medicamentos, foram incluídas no inquérito do sistema de Vigilância de Fatores de Risco e Proteção para Doenças Crônicas por Inquérito Telefônico (VIGITEL).

O objetivo do presente trabalho consistiu em analisar as diferenças sociodemográficas e regionais quanto ao acesso e fontes de obtenção de medicamentos, por indivíduos hipertensos e diabéticos com 20 anos ou mais de idade, residentes nas capitais brasileiras e no Distrito Federal, que são as áreas cobertas pelo inquérito VIGITEL.

\section{Método}

Trata-se de estudo transversal de base populacional que utilizou dados referentes à população de 20 anos ou mais, coletados em 2011, pelo VIGITEL, que é realizado anualmente nas capitais brasileiras e no Distrito Federal. O sistema VIGITEL estabelece um tamanho amostral mínimo de 2 mil indivíduos, com 18 ou mais anos de idade, em cada cidade. A seleção amostral é realizada em dois estágios: (1) sorteio de $5 \mathrm{mil}$ linhas telefônicas em cada cidade, seguido por 
novo sorteio para organização de 25 réplicas de 200 linhas; (2) sorteio de um morador adulto do domicílio com idade $\geq 18$ anos para responder à entrevista. Detalhes metodológicos do inquérito telefônico podem ser encontrados em publicações anteriores 14 .

As entrevistas telefônicas foram realizadas entre os meses de janeiro e dezembro de 2011. As perguntas abordaram características demográficas e socioeconômicas, informações sobre hábito alimentar e atividade física, peso e altura, frequência do consumo de cigarros e de bebidas alcoólicas, autoavaliação do estado de saúde, referência a diagnóstico médico anterior de algumas doenças e uso e fonte de obtenção de medicamentos para diabetes e hipertensão, dentre outros tópicos.

As variáveis analisadas neste trabalho foram: o relato de diabetes ou de hipertensão diagnosticada por algum médico o uso de medicamentos (para os que apresentavam estas doenças) e a fonte para obtê-los. Esses dados foram obtidos com base nas seguintes perguntas: "Algum médico já lhe disse que o(a) sr(a) tem pressão alta?", "Algum médico já lhe disse que o(a) sr(a) tem diabetes?", ambas categorizadas em "sim”, "não" e "não lembra" e "Onde o(a) sr(a) consegue a medicação para controlar a pressão alta? ou “...para o diabetes?", tendo por alternativas de resposta: unidade de saúde do SUS (US); PFPB; outro lugar; não sabe e não quis responder.

A categoria "outro lugar" resultou da junção das seguintes fontes de obtenção de medicamentos: drogarias e/ou farmácias não vinculadas ao PFPB e outras fontes de obtenção, tais como amostra gratuita recebida em consultórios médicos.

A prevalência de hipertensão e diabetes, os percentuais dos doentes em tratamento medicamentoso, bem como as fontes de obtenção dos medicamentos (de indivíduos hipertensos e diabéticos em tratamento) foram analisados segundo variáveis demográficas, socioeconômicas e local de residência:

Demográficas: sexo (feminino e masculino); faixas etárias (20-29, 30-39, 40-49, 50-59, 60-69, 70 ou mais anos); situação conjugal (casado/unido, solteiro, separado/divorciado e viúvo) e cor da pele (branca, preta, amarela, parda, indígena).

Socioeconômicas: escolaridade em anos de estudos (0-8, 9-11, 12 ou mais) e filiação ou não a plano médico de saúde.

Local de residência: capitais brasileiras e Distrito Federal.

Foram estimadas as prevalências de hipertensão e diabetes, os percentuais dos indivíduos em tratamento e as prevalências das diferentes fontes de obtenção de medicamentos, segundo as variáveis sociodemográficas, na população adulta (20 anos e mais). As razões de prevalência e respectivos intervalos de $95 \%$ de confiança (IC95\%), para cada fonte considerada, foram estimados utilizando-se regressão de Poisson 15. Também foram estimadas as frequências relativas percentuais, pontuais e por IC95\%, das fontes de obtenção dos medicamentos para as capitais e Distrito Federal. As análises foram realizadas com o uso do programa Stata 11 (StataCorp LP, College Station, Estados Unidos), o que permite considerar as ponderações relacionadas ao delineamento amostral complexo.

O projeto do sistema VIGITEL foi aprovado pela Comissão Nacional de Ética em Pesquisa para Seres Humanos do Ministério da Saúde (CONEP no 749/2006 - registro 13081; Atualização do Parecer no 355.590/2013). A assinatura do Termo de Consentimento Livre e Esclarecido, nesta pesquisa, foi substituída pelo consentimento verbal do entrevistado no momento da ligação telefônica que foi realizada por uma central e teve todas as entrevistas gravadas objetivando o controle de qualidade dos dados.

\section{Resultados}

Entre os 52.036 indivíduos pesquisados, 15.027 referiram hipertensão (24,3\%; IC95\%: 24,3-25,2) e 4.083 mencionaram ter diabetes mellitus $(6 \%$; IC95\%: 5,6-6,5). Faziam uso de medicamento $11.955(71,8 \%)$ dos hipertensos e $3.233(78,2 \%)$ dos diabéticos.

A Tabela 1 apresenta a prevalência de hipertensos e diabéticos e o percentual de indivíduos em tratamento medicamentoso segundo características sociodemográficas. Verifica-se maior prevalência de hipertensão arterial entre mulheres, viúvos e pessoas com menor escolaridade. Entre os diabéticos, observa-se também maior prevalência da doença nos viúvos, naqueles que referiram até 8 anos de estudos e, no limiar da significância estatística, entre os que possuíam plano de saúde. Para ambas as doenças, as prevalências apresentaram tendências ao aumento com o avanço da idade. Após ajuste por idade e sexo, a categoria "viúvos" perdeu a significância para ambas as doenças (dados não apresentados em tabela).

A análise das razões de prevalência ajustadas por idade e sexo permitiu verificar que as menores prevalências de hipertensos em tratamento medicamentoso ocorreram entre os homens, nos indivíduos entre 20-29 anos, nos solteiros e viúvos, nos com menos de 12 anos de escolaridade e entre os que não possuíam plano de saúde. Entre diabéticos, verificaram-se menores prevalências 
Tabela 1

Prevalência de hipertensos e diabéticos e percentual em tratamento medicamentoso, segundo características sociodemográficas. Vigilância de Fatores de Risco e Proteção para Doenças Crônicas por Inquérito Telefônico (VIGITEL).

\begin{tabular}{|c|c|c|c|c|c|c|}
\hline Variável & $\begin{array}{l}\text { Prevalência de } \\
\text { hipertensos * } \\
(n=15.027)\end{array}$ & $\begin{array}{l}\text { Percentual de } \\
\text { hipertensos em } \\
\text { tratamento } \\
(n=11.955)\end{array}$ & $\begin{array}{l}\text { RP ajustada } \\
\text { (IC95\%) }\end{array}$ & $\begin{array}{l}\text { Prevalência de } \\
\text { diabéticos * } \\
(n=4.083)\end{array}$ & $\begin{array}{l}\text { Percentual de } \\
\text { diabéticos em } \\
\text { tratamento } \\
(\mathrm{n}=3.233)\end{array}$ & $\begin{array}{l}\text { RP ajustada } \\
\text { (IC95\%) }\end{array}$ \\
\hline & $\%$ & \% (IC95\%) & & $\%$ & \% (IC95\%) & \\
\hline Sexo & $<0,001$ & $<0,001$ & & 0,124 & 0,846 & \\
\hline Masculino & 21,2 & $64,2(60,8-67,4)$ & 1,00 & 5,7 & $78,6(72,0-83,9)$ & 1,00 \\
\hline Feminino & 27,1 & $76,9(74,0-80,0)$ & $1,14(1,08-1,20)$ & 6,4 & $77,9(74,4-81,0)$ & $0,98(0,91-1,06)$ \\
\hline Idade (anos) & $<0,001$ & $<0,001$ & & $<0,001$ & $<0,001$ & \\
\hline $20-29$ & 7,8 & $18,1(11,3-27,8)$ & 1,00 & 0,8 & $15,0(5,4-35,3)$ & 1,00 \\
\hline $30-39$ & 14,1 & $47,0(41,6-52,6)$ & $2,57(1,61-4,11)$ & 1,6 & $61,6(49,3-72,6)$ & $4,1(1,56-11,02)$ \\
\hline $40-49$ & 25,7 & $66,7(62,8-70,4)$ & $3,67(2,32-5,78)$ & 5,5 & $77,7(70,6-83,5)$ & $5,2(1,99-13,66)$ \\
\hline $50-59$ & 41,5 & $83,5(81,0-85,7)$ & $4,57(2,90-7,19)$ & 11,7 & $80,5(76,1-84,3)$ & $5,4(2,06-14,09)$ \\
\hline $60-69$ & 57,1 & $90,9(88,3-93,0)$ & $4,95(3,14-7,80)$ & 18,8 & $85,1(81,0-88,4)$ & $5,7(2,19-14,94)$ \\
\hline 70 ou mais & 60,3 & $95,6(94,3-96,6)$ & $5,17(3,28-8,15)$ & 22,0 & $84,6(79,3-88,7)$ & $5,7(2,17-14,83)$ \\
\hline Cor & 0,185 & 0,006 & & 0,381 & 0,489 & \\
\hline Branca & 25,2 & $75,1(71,9-78,1)$ & 1,00 & 6,3 & $73,2(63,3-81,2)$ & 1,00 \\
\hline Preta/Parda & 23,5 & $69,4(66,1-72,4)$ & $0,99(0,94-1,04)$ & 5,9 & $78,7(74,0-82,7)$ & $1,04(0,96-1,13)$ \\
\hline Amarela & 23,5 & $72,6(62,2-81,1)$ & $0,92(0,81-1,04)$ & 6,3 & $83,4(78,5-87,3)$ & $1,08(0,93-1,25)$ \\
\hline Indígena & 21,3 & $58,8(44,6-71,7)$ & $0,89(0,71-1,11)$ & 4,1 & $75,6(67,1-82,5)$ & $0,84(0,54-1,29)$ \\
\hline Situação conjugal & $<0,001$ & $<0,001$ & & $<0,001$ & 0,196 & \\
\hline Solteiro & 14,7 & $52,1(46,3-57,9)$ & 1,00 & 2,7 & $73,2(63,3-81,2)$ & 1,00 \\
\hline Casado/Unido & 27,5 & $74,2(71,9-76,3)$ & $1,18(1,08-1,28)$ & 7,1 & $78,7(74,0-82,7)$ & $0,99(0,88-1,13)$ \\
\hline Viúvo & 56,3 & $92,1(89,2-94,2)$ & $1,01(0,93-1,09)$ & 17,2 & $83,4(78,5-87,3)$ & $0,93(0,81-1,06)$ \\
\hline Separado/ & 31,7 & $83,8(79,7-87,1)$ & $1,22(1,11-1,33)$ & 9,0 & $75,6(67,1-82,5)$ & $0,96(0,83-1,11)$ \\
\hline \multicolumn{7}{|l|}{ Divorciado } \\
\hline Escolaridade (anos) & $<0,001$ & $<0,001$ & & $<0,001$ & 0,039 & \\
\hline $0-8$ & 30,0 & $73,8(70,6-76,7)$ & 1,00 & 8,0 & $78,6(74,2-82,5)$ & 1,00 \\
\hline $9-11$ & 17,4 & $63,3(60,2-66,2)$ & $1,01(0,95-1,08)$ & 3,7 & $72,6(66,8-77,8)$ & $0,99(0,89-1,09)$ \\
\hline 12 ou mais & 17,9 & $75,2(71,8-78,3)$ & $1,14(1,08-1,21)$ & 3,8 & $84,0(78,2-88,4)$ & $1,10(1,01-1,21)$ \\
\hline Plano de saúde & 0,603 & $<0,001$ & & 0,051 & $<0,001$ & \\
\hline Possui & 24,0 & $78,0(75,7-80,2)$ & 1,00 & 6,5 & $83,5(80,2-86,4)$ & 1,00 \\
\hline Não possui & 24,5 & $67,4(64,1-70,5)$ & $0,95(0,92-0,99)$ & 5,7 & $73,7(68,3-78,4)$ & $0,93(0,87-0,99)$ \\
\hline
\end{tabular}

IC95\%: intervalo de 95\% de confiança; RP ajustada: razão de prevalência ajustada por sexo e idade.

* Prevalências calculadas sob ponderações devidas ao desenho amostral.

de tratamento nos indivíduos mais jovens e naqueles com escolaridade inferior a 12 anos e nos que não possuíam plano de saúde.

A Tabela 2 apresenta a prevalência e razão de prevalência das diferentes fontes de obtenção de medicamentos dos pacientes hipertensos, segundo características sociodemográficas. Após ajuste por sexo e idade, as razões de prevalência apontam que as maiores prevalências de obtenção nas US foram observadas nas mulheres, nos indivíduos com idade inferior a 70 anos, nos que referiram cor de pele preta ou parda, naqueles com escolaridade até 8 anos de estudos e nos que não possuíam plano privado de saúde. Esses foram os segmentos da população que apresentaram as menores prevalências de obtenção de medicamentos para hipertensão nas drogarias e farmácias não vinculadas ao PFPB e em outras fontes.

No PFPB, menores prevalências de obtenção foram observadas entre pretos/pardos e naqueles que não possuíam plano de saúde, e maior nos indivíduos com 9 ou mais anos de estudos.

A Tabela 3 apresenta a prevalência e razão de prevalência das diferentes fontes de obtenção de medicamentos pelos diabéticos, segundo 
Prevalência e razão de prevalência de fontes de obtenção de medicamentos, por indivíduos hipertensos, segundo características sociodemográficas. Vigilância de Fatores de Risco e Proteção para Doenças Crônicas por Inquérito Telefônico (VIGITEL)

\begin{tabular}{|c|c|c|c|c|c|c|c|c|c|}
\hline \multirow[t]{2}{*}{ Variável } & \multicolumn{3}{|c|}{ US } & \multicolumn{3}{|c|}{ PFPB } & \multicolumn{3}{|c|}{ Drogarias e/ou farmácias e outros * } \\
\hline & $\mathrm{n}$ & $\%$ & $\begin{array}{l}\text { RP ajustada } \\
\text { (IC95\%) }\end{array}$ & $\mathrm{n}$ & $\%$ & $\begin{array}{l}\text { RP ajustada } \\
\text { (IC95\%) }\end{array}$ & $\mathbf{n}$ & $\%$ & $\begin{array}{l}\text { RP ajustada } \\
\text { (IC95\%) }\end{array}$ \\
\hline \multicolumn{10}{|l|}{ Sexo } \\
\hline Masculino & 1.154 & 42,5 & 1,00 & 616 & 15,8 & 1,00 & 2.055 & 41,7 & 1,00 \\
\hline Feminino & 2.946 & 47,7 & $1,13(1,03-1,24)$ & 1.285 & 15,9 & $1,00(0,84-1,20)$ & 3.876 & 36,4 & $0,86(0,79-0,95)$ \\
\hline Total & 4.100 & 45,8 & & 1.901 & 15,9 & & 5.931 & 38,3 & \\
\hline \multicolumn{10}{|l|}{ Idade (anos) } \\
\hline $20-39$ & 238 & 51,9 & $1,48(1,23-1,77)$ & 125 & 15,8 & $0,89(0,64-1,24)$ & 338 & 32,2 & $0,68(0,55-0,85)$ \\
\hline $40-49$ & 591 & 46,8 & $1,34(1,16-1,55)$ & 281 & 15,8 & $0,89(0,68-1,15)$ & 834 & 37,4 & $0,79(0,68-0,91)$ \\
\hline $50-59$ & 1.165 & 47,4 & $1,36(1,19-1,54)$ & 470 & 15,6 & $0,88(0,70-1,10)$ & 1.563 & 37,0 & $0,78(0,69-0,88)$ \\
\hline $60-69$ & 1.217 & 49,3 & $1,40(1,24-1,59)$ & 543 & 14,5 & $0,81(0,65-1,01)$ & 1.625 & 36,2 & $0,77(0,68-0,86)$ \\
\hline 70 ou mais & 889 & 35,2 & 1,00 & 482 & 17,8 & 1,00 & 1.571 & 47,0 & 1,00 \\
\hline \multicolumn{10}{|l|}{ Cor } \\
\hline Branca & 1.529 & 39,9 & 1,00 & 929 & 18,0 & 1,00 & 2.902 & 42,1 & 1,00 \\
\hline Preta/Parda & 2.286 & 50,9 & $1,26(1,14-1,38)$ & 845 & 13,6 & $0,76(0,64-0,90)$ & 2.773 & 35,5 & $0,86(0,78-0,94)$ \\
\hline Amarela & 98 & 43,5 & $1,08(0,79-1,48)$ & 49 & 15,3 & $0,84(0,48-1,47)$ & 114 & 41,2 & $0,99(0,71-1,38)$ \\
\hline Indígena & 69 & 52,8 & $1,29(0,88-1,88)$ & 25 & 26,7 & $1,50(0,79-2,85)$ & 60 & 20,5 & $0,50(0,23-1,09)$ \\
\hline \multicolumn{10}{|l|}{ Situação conjugal } \\
\hline Solteiro & 663 & 48,2 & 1,00 & 296 & 15,8 & 1,00 & 881 & 35,9 & 1,00 \\
\hline Casado/Unido & 2.142 & 45,9 & $1,02(0,91-1,16)$ & 1.039 & 15,4 & $0,95(0,74-1,24)$ & 3.229 & 38,6 & $0,99(0,86-1,14)$ \\
\hline Viúvo & 813 & 42,6 & $1,00(0,86-1,18)$ & 350 & 18,2 & $1,10(0,81-1,51)$ & 1.177 & 39,2 & $0,95(0,80-1,13)$ \\
\hline Separado/Divorciado & 464 & 47,1 & $1,04(0,88-1,22)$ & 209 & 14,8 & $0,92(0,65-1,30)$ & 611 & 38 & $0,99(0,82-1,20)$ \\
\hline \multicolumn{10}{|l|}{ Escolaridade (anos) } \\
\hline $0-8$ & 2.820 & 55,2 & 1,00 & 824 & 14,5 & 1,00 & 2.103 & 30,4 & 1,00 \\
\hline $9-11$ & 992 & 33,8 & $0,56(0,50-0,63)$ & 594 & 19,1 & $1,37(1,14-1,66)$ & 1.905 & 47,1 & $1,70(1,52-1,88)$ \\
\hline 12 ou mais & 288 & 14,5 & $0,25(0,19-0,31)$ & 483 & 18,8 & $1,35(1,08-1,69)$ & 1.923 & 66,7 & $2,34(2,11-2,60)$ \\
\hline \multicolumn{10}{|l|}{ Plano de saúde } \\
\hline Possui & 1.060 & 20,8 & 1,00 & 1.271 & 23,3 & 1,00 & 4.306 & 55,9 & 1,00 \\
\hline Não possui & 3.036 & 66,4 & $3,16(2,80-3,57)$ & 629 & 9,8 & $0,42(0,35-0,50)$ & 1.614 & 23,9 & $0,43(0,39-0,48)$ \\
\hline
\end{tabular}

IC95\%: intervalo de 95\% de confiança; PFPB: Programa Farmácia Popular do Brasil; RP ajustada: razão de prevalência ajustada por sexo e idade; US: unidade de saúde do SUS.

* Drogarias e farmácias não credenciadas ao PFPB.

características sociodemográficas. A obtenção nas US, após ajuste por idade e sexo, apresentou prevalências significativamente maiores nas idades inferiores a 70 anos, nos indivíduos de cor da pele preta ou parda e nos que referiram não possuir plano de saúde. Entre os viúvos e naqueles com 9 ou mais anos de estudos, as prevalências foram menores.

A obtenção de medicamentos para diabetes no PFPB, após ajuste, apresentou prevalências significativamente maiores entre aqueles que possuem 9 ou mais anos de estudos. Nos indivíduos com 20 a 39 anos, nos respondentes de cor preta/parda, e entre os que não possuíam plano de saúde, as prevalências foram menores.

Em relação aos indivíduos que referiram obter medicamentos para diabetes em drogarias e/ ou farmácias e outras fontes, após o ajuste por idade e sexo, observaram-se maiores prevalências entre os mais escolarizados e menores prevalências nos entrevistados de cor preta/parda, naqueles com idades entre 40 e 69 anos e entre os que não possuíam plano de saúde.

Quanto às fontes de obtenção de medicamentos, $45,8 \%$ dos hipertensos os conseguiram nas US; $15,9 \%$ no PFPB; e $38,3 \%$ em drogarias e 
Tabela 3

Prevalência e razão de prevalência de fontes de obtenção de medicamentos, por indivíduos diabéticos, segundo características sociodemográficas. Vigilância de Fatores de Risco e Proteção para Doenças Crônicas por Inquérito Telefônico (VIGITEL)

\begin{tabular}{|c|c|c|c|c|c|c|c|c|c|}
\hline \multirow[t]{2}{*}{ Variável } & \multicolumn{3}{|c|}{ US } & \multicolumn{3}{|c|}{ PFPB } & \multicolumn{3}{|c|}{ Drogarias e/ou farmácias e outros * } \\
\hline & $\mathbf{n}$ & $\%$ & $\begin{array}{l}\text { RP ajustada } \\
\text { (IC95\%) }\end{array}$ & $\mathbf{n}$ & $\%$ & $\begin{array}{l}\text { RP ajustada } \\
\text { (IC95\%) }\end{array}$ & $\mathrm{n}$ & $\%$ & $\begin{array}{l}\text { RP ajustada } \\
\text { (IC95\%) }\end{array}$ \\
\hline \multicolumn{10}{|l|}{ Sexo } \\
\hline Masculino & 466 & 51,5 & 1,00 & 191 & 15,8 & 1,00 & 509 & 32,7 & 1,00 \\
\hline Feminino & 997 & 56,6 & $1,10(0,96-1,26)$ & 352 & 16,5 & $1,04(0,75-1,44)$ & 706 & 26,9 & $0,82(0,67-1,01)$ \\
\hline Total & 1.663 & 54,4 & & & & & & & \\
\hline \multicolumn{10}{|l|}{ Idade (anos) } \\
\hline $20-39$ & 57 & 59,8 & $1,48(1,11-1,98)$ & 22 & 8,9 & $0,50(0,25-0,98)$ & 50 & 31,3 & $0,75(0,48-1,16)$ \\
\hline $40-49$ & 186 & 66,6 & $1,65(1,31-2,07)$ & 75 & 16,2 & $0,91(0,53-1,57)$ & 109 & 17,3 & $0,41(0,27-0,63)$ \\
\hline $50-59$ & 395 & 58,4 & $1,45(1,17-1,81)$ & 132 & 16,0 & $0,90(0,59-1,36)$ & 280 & 25,6 & $0,61(0,45-0,81)$ \\
\hline $60-69$ & 492 & 53,1 & $1,31(1,05-1,62)$ & 174 & 16,8 & $0,94(0,62-1,44)$ & 388 & 30,1 & $0,73(0,57-0,93)$ \\
\hline 70 ou mais & 333 & 40,3 & 1,00 & 140 & 17,8 & 1,00 & 388 & 41,9 & 1,00 \\
\hline \multicolumn{10}{|l|}{ Cor } \\
\hline Branca & 530 & 43,3 & 1,00 & 267 & 221 & 1,00 & 605 & 34,6 & 1,00 \\
\hline Preta/Parda & 817 & 62,5 & $1,40(1,21-1,62)$ & 239 & 11,8 & $0,54(0,39-0,75)$ & 559 & 25,7 & $0,78(0,63-0,96)$ \\
\hline Amarela & 39 & 54,2 & $1,26(0,89-1,80)$ & 15 & 11,1 & $0,50(0,24-1,02)$ & 27 & 34,7 & $0,97(0,59-1,61)$ \\
\hline Indígena & 28 & 64,4 & $1,41(0,89-2,23)$ & 5 & 19,1 & $0,87(0,19-4,05)$ & 10 & 16,5 & $0,54(0,20-1,45)$ \\
\hline \multicolumn{10}{|l|}{ Situação conjugal } \\
\hline Solteiro & 226 & 61,8 & 1,00 & 82 & 15,2 & 1,00 & 158 & 23,0 & 1,00 \\
\hline Casado/Unido & 763 & 56,6 & $1,03(0,86-1,23)$ & 290 & 14,8 & $0,94(0,56-1,58)$ & 681 & 28,6 & $1,00(0,69-1,45)$ \\
\hline Viúvo & 316 & 40,4 & $0,78(0,61-0,98)$ & 113 & 23,5 & $1,55(0,89-2,71)$ & 253 & 36,2 & $1,14(0,77-1,69)$ \\
\hline Separado/Divorciado & 155 & 49,2 & $0,85(0,65-1,11)$ & 56 & 14,7 & $0,96(0,48-1,88)$ & 118 & 36,1 & $1,41(0,91-2,18)$ \\
\hline \multicolumn{10}{|l|}{ Escolaridade (anos) } \\
\hline $0-8$ & 990 & 62,1 & 1,00 & 226 & 13,7 & 1,00 & 472 & 24,3 & 1,00 \\
\hline $9-11$ & 350 & 45,7 & $0,65(0,56-0,76)$ & 180 & 18,7 & $1,46(1,01-2,11)$ & 363 & 35,6 & $1,71(1,36-2,15)$ \\
\hline 12 ou mais & 123 & 19,7 & $0,29(0,20-0,42)$ & 137 & 28,2 & $2,23(1,53-3,26)$ & 380 & 52,1 & $2,34(2,86-2,95)$ \\
\hline \multicolumn{10}{|l|}{ Plano de saúde } \\
\hline Possui & 488 & 32,4 & 1,00 & 365 & 20,9 & 1,00 & 923 & 46,7 & 1,00 \\
\hline Não possui & 974 & 75,1 & $2,24(1,88-2,66)$ & 176 & 11,7 & $0,56(0,40-0,79)$ & 290 & 13,2 & $0,30(0,24-0,38)$ \\
\hline
\end{tabular}

IC95\%: intervalo de 95\% de confiança; PFPB: Programa Farmácia Popular do Brasil; RP ajustada: razão de prevalência ajustada por sexo e idade; US: unidade de saúde do SUS.

* Drogarias e farmácias não credenciadas ao PFPB.

farmácias não credenciadas ao programa e outras fontes (drogarias e/ou farmácias e outros). Entre os diabéticos, esses percentuais foram de $54,4 \%, 16,2 \%$ e $29,4 \%$, respectivamente (dados apresentados nas Tabelas 2 e 3).

No tocante ao conjunto das capitais brasileiras (Tabela 4), menores percentuais de obtenção dos medicamentos para hipertensão, nas US, foram observados nas cidades de Natal, Rio Grande do Norte (16,2\%), São Luís, Maranhão (16,2\%) e Belém, Pará (26,8\%), e os maiores em São Paulo (59,7\%), Vitória, Espírito Santo (56,1\%) e Rio Branco, Acre (54,5\%). Em relação à obtenção do medicamento para hipertensão por meio do PFPB, os menores percentuais ocorreram em Curitiba, Paraná $(7,9 \%)$, Cuiabá, Mato Grosso (9\%) e Rio Branco $(9,8)$, sendo que os maiores percentuais foram registrados em Natal $(25,1 \%)$, Belém (25\%) e Rio de Janeiro (24,9\%).

Quanto à obtenção de medicamentos para hipertensão em drogarias e/ou farmácias não credenciadas ao PFPB - situação em que os pacientes tiveram de arcar com o custo total dos medicamentos - maiores percentuais foram verificados nas capitais Natal (58,7\%), São Luís $(58,1 \%)$ e Goiânia, Goiás $(56,3 \%)$ e os menores em São Paulo (29,6\%), Belo Horizonte, Minas Gerais $(30,9 \%)$ e Vitória $(32,6 \%)$. 
Tabela 4

Distribuição percentual de adultos (20 anos ou mais) que referiram conseguir medicamento para controle da hipertensão arterial e diabetes, segundo formas de obtenção nas regiões e capitais brasileiras. Vigilância de Fatores de Risco e Proteção para Doenças Crônicas por Inquérito Telefônico (VIGITEL).

\begin{tabular}{|c|c|c|c|c|c|c|c|}
\hline \multirow[t]{3}{*}{ Regiões/Capitais } & \multicolumn{7}{|c|}{ Hipertensão } \\
\hline & \multirow{2}{*}{$\begin{array}{c}\text { Total } \\
\text { n }\end{array}$} & \multicolumn{2}{|r|}{ US } & \multicolumn{2}{|c|}{ PFPB } & \multicolumn{2}{|c|}{ Drogarias e farmácias e outros * } \\
\hline & & $\%$ & IC95\% & $\%$ & IC95\% & $\%$ & IC95\% \\
\hline Centro-oeste & 1.853 & 44,9 & $41,20-48,60$ & 12,1 & $10,10-4,60$ & 42,6 & $39,10-46,10$ \\
\hline Campo Grande & 497 & 52,1 & $45,70-58,50$ & 14,1 & $7,40-20,70$ & 33,8 & $28,40-39,20$ \\
\hline Cuiabá & 439 & 48,4 & $40,70-56,00$ & 9,0 & $5,40-12,50$ & 42,7 & $35,50-49,70$ \\
\hline Goiânia & 459 & 27,3 & $22,20-32,30$ & 16,4 & $12,30-20,50$ & 56,3 & $50,70-61,90$ \\
\hline Distrito Federal & 457 & 51,3 & $44,90-57,60$ & 10,5 & $7,150-13,80$ & 38,2 & $32,20-44,20$ \\
\hline Nordeste & 4.093 & 40,7 & $38,20-43,10$ & 14,9 & $13,00-16,70$ & 44,4 & $42,00-46,80$ \\
\hline Aracaju & 444 & 41,0 & $33,00-49,00$ & 14,0 & $8,40-19,60$ & 45,0 & $37,70-52,20$ \\
\hline Fortaleza & 371 & 51,1 & $43,90-58,30$ & 15,1 & $9,20-21,10$ & 33,7 & $27,30-40,10$ \\
\hline João Pessoa & 514 & 40,7 & $34,00-47,30$ & 14,9 & $10,10-19,60$ & 44,5 & $38,10-50,80$ \\
\hline Maceió & 474 & 39,6 & $10,90-21,50$ & 14,4 & $9,93-18,90$ & 46,0 & $39,90-52,00$ \\
\hline Natal & 459 & 16,2 & $10,90-21,50$ & 25,1 & $18,50-31,60$ & 58,7 & $50,60-66,80$ \\
\hline Recife & 599 & 42,0 & $36,20-47,70$ & 15,3 & $11,40-19,10$ & 42,7 & $37,00-48,50$ \\
\hline Salvador & 454 & 41,5 & $35,50-47,40$ & 11,9 & $8,02-15,70$ & 46,6 & $40,80-52,40$ \\
\hline São Luís & 357 & 25,8 & $18,20-33,40$ & 16,1 & $11,10-21,10$ & 58,1 & $50,30-65,80$ \\
\hline Teresina & 422 & 49,0 & $41,40-56,50$ & 11,5 & $7,40-15,50$ & 39,5 & $32,50-46,50$ \\
\hline Norte & 2.363 & 38,2 & $34,20-42,10$ & 18,5 & $15,30-21,70$ & 43,3 & $39,50-47,00$ \\
\hline Belém & 370 & 26,8 & $20,40-33,50$ & 25,0 & $13,70-24,30$ & 48,3 & $41,40-55,10$ \\
\hline Boa Vista & 331 & 46,1 & $36,60-55,40$ & 15,5 & $10,00-21,00$ & 38,4 & $29,90-46,80$ \\
\hline Macapá & 342 & 26,8 & $18,80-34,70$ & 17,6 & $7,60-27,60$ & 55,6 & $45,70-65,50$ \\
\hline Manaus & 351 & 49,2 & $41,20-57,20$ & 14,4 & $8,30-20,40$ & 36,4 & $29,10-43,60$ \\
\hline Palmas & 277 & 30,2 & $21,00-39,30$ & 23,8 & $15,90-31,60$ & 46,0 & $36,40-55,50$ \\
\hline Rio Branco & 398 & 54,5 & $47,70-61,20$ & 9,8 & $5,50-4,10$ & 35,7 & $29,30-41,20$ \\
\hline Porto Velho & 294 & 42,3 & $34,50-49,90$ & 13,1 & $8,50-17,60$ & 44,7 & $37,10-52,10$ \\
\hline Sudeste & 2.082 & 48,8 & $45,30-52,30$ & 17,3 & $14,90-19,60$ & 33,9 & $30,70-37,00$ \\
\hline Belo Horizonte & 502 & 49,6 & $43,90-55,20$ & 19,5 & $15,40-23,60$ & 30,9 & $20,90-31,90$ \\
\hline São Paulo & 434 & 59,7 & $54,20-65,00$ & 10,8 & $7,50-13,90$ & 29,6 & $24,60-34,50$ \\
\hline Vitória & 522 & 56,1 & $50,60-61,60$ & 11,3 & $8,20-14,40$ & 32,6 & $27,60-37,50$ \\
\hline Rio de Janeiro & 624 & 35,0 & $29,30-40,60$ & 24,9 & $20,50-29,20$ & 40,2 & $35,20-45,10$ \\
\hline Sul & 1.541 & 49,6 & $45,80-53,40$ & 11,3 & $9,20-13,40$ & 39,0 & $35,40-42,60$ \\
\hline Curitiba & 473 & 49,2 & $43,20-55,20$ & 7,9 & $5,50-10,30$ & 42,9 & $31,70-42,10$ \\
\hline Florianópolis & 504 & 47,2 & $41,60-52,80$ & 15,9 & $11,80-19,90$ & 36,9 & $31,70-42,10$ \\
\hline Porto Alegre & 564 & 50,4 & $44,70-56,00$ & 13,8 & $10,10-17,50$ & 35,8 & $30,70-40,80$ \\
\hline Total & 11.932 & & & & & & \\
\hline
\end{tabular}

(continua) 
Tabela 4 (continuação)

\begin{tabular}{|c|c|c|c|c|c|c|c|}
\hline \multirow[t]{3}{*}{ Regiões/Capitais } & \multicolumn{7}{|c|}{ Diabetes } \\
\hline & \multirow{2}{*}{$\begin{array}{c}\text { Total } \\
\text { n }\end{array}$} & \multicolumn{2}{|r|}{ US } & \multicolumn{2}{|c|}{ PFPB } & \multicolumn{2}{|c|}{ Drogarias e farmácias e outros * } \\
\hline & & $\%$ & IC95\% & $\%$ & IC95\% & $\%$ & IC95\% \\
\hline Centro-oeste & 461 & 54,8 & $47,70-61,90$ & 12,1 & $7,80-16,40$ & 33,1 & $26,50-39,60$ \\
\hline Campo Grande & 131 & 61,0 & $49,80-72,20$ & 9,0 & $3,20-14,70$ & 30,0 & $19,10-40,80$ \\
\hline Cuiabá & 109 & 54,8 & $42,30-67,30$ & 15,7 & $4,30-26,90$ & 29,5 & $19,10-39,90$ \\
\hline Goiânia & 100 & 43,1 & $30,50-55,60$ & 17,7 & $30,50-55,60$ & 39,3 & $8,90-26,40$ \\
\hline Distrito Federal & 121 & 57,5 & $45,70-69,40$ & 10,2 & $45,70-69,30$ & 32,2 & $21,40-43,00$ \\
\hline Nordeste & 1.166 & 53,7 & $49,10-58,40$ & 12,2 & $9,50-14,90$ & 34,1 & $29,80-38,30$ \\
\hline Aracaju & 131 & 58,3 & $46,80-69,80$ & 8,7 & $2,40-14,80$ & 33,0 & $22,40-43,50$ \\
\hline Fortaleza & 127 & 59,2 & $46,90-71,40$ & 11,2 & $3,40-19,00$ & 29,6 & $18,40-40,70$ \\
\hline João Pessoa & 140 & 60,3 & $49,50-70,90$ & 11,0 & $3,70-18,20$ & 28,8 & $19,80-37,60$ \\
\hline Maceió & 141 & 57,5 & $46,70-68,10$ & 10,8 & $4,58-17,10$ & 31,7 & $22,00-41,30$ \\
\hline Natal & 139 & 19,5 & $10,50-28,50$ & 29,4 & $18,80-39,90$ & 51,1 & $39,60-62,50$ \\
\hline Recife & 156 & 48,1 & $38,20-58,00$ & 17,7 & $10,40-24,80$ & 34,2 & $25,20-43,20$ \\
\hline Salvador & 122 & 59,8 & $47,70-71,80$ & 5,4 & $1,80-8,90$ & 34,8 & $23,30-46,30$ \\
\hline São Luís & 116 & 50,0 & $36,70-63,30$ & 12,6 & $5,40-19,80$ & 37,4 & $25,40-49,20$ \\
\hline Teresina & 94 & 61,1 & $49,20-72,90$ & 8,1 & $1,70-14,50$ & 30,8 & $20,00-41,50$ \\
\hline Norte & 648 & 52,5 & $45,50-59,50$ & 19,0 & $13,70-24,30$ & 28,5 & $22,80-34,20$ \\
\hline Belém & 113 & 47,2 & $34,00-60,20$ & 27,3 & $16,30-38,30$ & 25,5 & $15,70-35,30$ \\
\hline Boa Vista & 92 & 48,9 & $30,20-67,60$ & 20,9 & $8,50-33,40$ & 30,1 & $14,00-46,30$ \\
\hline Macapá & 90 & 32,3 & $14,70-49,80$ & 13,8 & $1,80-25,80$ & 53,9 & $34,90-72,80$ \\
\hline Manaus & 105 & 63,2 & $51,50-74,80$ & 11,7 & $4,50-18,90$ & 25,1 & $15,20-35,00$ \\
\hline Palmas & 72 & 49,8 & $27,80-71,80$ & 22,0 & $7,14-36,70$ & 28,2 & $12,00-44,40$ \\
\hline Rio Branco & 94 & 63,0 & $49,00-76,90$ & 5,3 & $1,07-9,40$ & 31,7 & $17,70-45,80$ \\
\hline Porto Velho & 82 & 51,5 & $37,60-65,30$ & 19,3 & $8,30-30,30$ & 29,2 & $17,10-41,20$ \\
\hline Sudeste & 534 & 54,6 & $47,90-61,20$ & 19,0 & $14,20-23,80$ & 26,5 & $21,00-31,90$ \\
\hline Belo Horizonte & 120 & 60,6 & $49,90-71,20$ & 17,5 & $9,80-25,10$ & 22,0 & $12,20-31,80$ \\
\hline São Paulo & 143 & 62,5 & $53,20-71,80$ & 15,0 & $8,30-21,60$ & 22,5 & $15,10-29,90$ \\
\hline Vitória & 131 & 69,5 & $60,10-78,90$ & 11,6 & $5,60-17,70$ & 18,8 & $11,30-26,40$ \\
\hline Rio de Janeiro & 140 & 37,0 & $26,20-47,80$ & 27,3 & $18,40-36,20$ & 35,7 & $25,50-45,90$ \\
\hline Sul & 412 & 57,0 & $49,90-64,10$ & 12,0 & $8,00-16,10$ & 30,9 & $24,00-37,80$ \\
\hline Curitiba & 120 & 56,8 & $45,20-68,40$ & 9,7 & $3,10-16,30$ & 33,5 & $21,80-45,10$ \\
\hline Florianópolis & 151 & 57,1 & $46,40-67,80$ & 9,5 & $4,40-14,60$ & 33,4 & $22,60-44,10$ \\
\hline Porto Alegre & 141 & 57,2 & $46,30-68,00$ & 14,6 & $8,40-20,80$ & 28,2 & $18,20-38,30$ \\
\hline Total & 3.221 & & & & & & \\
\hline
\end{tabular}

IC95\%: intervalo de 95\% de confiança; PFPB: Programa Farmácia Popular do Brasil; RP ajustada: razão de prevalência ajustada por sexo e idade; US: unidade de saúde do SUS.

* Drogarias e farmácias não credenciadas ao PFPB.

Para o diabetes, os menores percentuais de obtenção dos medicamentos em US foram registrados em Natal (19,5\%), Macapá, Amapá (32,3\%) e Rio de Janeiro (37\%), e os maiores em Vitória (69,5\%), Manaus, Amazonas (63,2\%), Rio Branco
(63\%) e São Paulo (62,5\%). Quanto à obtenção por meio do PFPB, os menores percentuais constaram em Rio Branco (5,3\%), Salvador, Bahia $(5,4 \%)$ e Teresina, Piauí $(8,1 \%)$, e os maiores em Belém (27,3\%), Rio de Janeiro (27,3\%) e Natal $(29,4 \%)$. 
A obtenção de medicamentos em drogarias e/ ou farmácias não credenciadas ao PFPB e outros locais foi menor nas capitais Vitória (18,8\%), Belo Horizonte (22\%) e São Paulo (22,5\%), e maior em Goiânia (39,3\%), Natal (51,1\%) e Macapá (53,9\%).

A Tabela 4 apresenta a distribuição percentual, nas cinco regiões brasileiras e capitais, dos adultos e idosos que obtiveram medicamentos para controle da hipertensão e do diabetes, segundo fontes de obtenção.

Para os hipertensos, foram observados maiores percentuais de obtenção em US das capitais, nas regiões Sul $(49,6 \%)$ e Sudeste $(48,8 \%)$; no PFPB, nas regiões Norte $(18,5 \%)$ e Sudeste (17,3\%); e em drogarias e/ou farmácias, nas regiões Nordeste $(44,4 \%)$ e Norte $(43,3 \%)$. Padrão similar foi observado em relação ao diabetes, com maiores percentuais de obtenção: em US, nas capitais do Sul $(49,6 \%)$ e Sudeste $(48,8 \%)$; nas farmácias do PFPB, nas regiões Norte $(18,5 \%)$ e Sudeste (17,3\%); e em drogarias e/ou farmácias não credenciadas ao PFPB e outros locais, nas regiões Nordeste $(44,4 \%)$ e Norte $(43,3 \%)$.

\section{Discussão}

As prevalências de hipertensão arterial e diabetes mellitus vêm crescendo no Brasil 4 e mundialmente, configurando-se como uma epidemia resultante, em grande parte, do envelhecimento populacional 16 e do processo de globalização dos riscos 2. Constatou-se, no presente trabalho, prevalências mais elevadas dessas doenças nos estratos de menor nível de escolaridade, como observado em estudos prévios desenvolvidos com dados das PNAD de 2003 e 20084 e do VIGITEL 14 .

A hipertensão e o diabetes configuram-se como fatores de risco para outras doenças cardiovasculares, cuja diminuição de mortalidade, constatada nas últimas décadas, vem sendo parcialmente atribuída ao controle adequado da pressão arterial 14,17,18. Em estudo sobre o perfil de utilização de medicamentos por aposentados brasileiros 5 , constatou-se que $88,7 \%$ dos idosos hipertensos utilizavam medicamentos anti-hipertensivos e $64,7 \%$ dos diabéticos usavam antidiabéticos. Neste estudo, foram encontrados em tratamento medicamentoso $71,8 \%$ dos hipertensos e $78,2 \%$ dos diabéticos. Para ambas as doenças os segmentos com as menores frequências de pacientes em tratamento foram os de indivíduos entre 20-29 anos, aqueles com menos de 12 anos de escolaridade e os que não possuíam plano de saúde. Para esses segmentos, dentre os quais se incluem pacientes usuários do SUS, preconizase ampliar as estratégias de orientação terapêu- tica medicamentosa nos serviços públicos de saúde, a fim de evitar prejuízos decorrentes da desassistência no tratamento farmacológico.

Frequências mais reduzidas de tratamento medicamentoso ou a não utilização de medicamentos, entre portadores de hipertensão e diabetes, podem estar relacionadas a barreiras no acesso e na adesão ao tratamento 5. É possível ainda considerar que alguns segmentos de pacientes estariam reduzindo a utilização de medicamentos e adotando outras formas de controle das doenças, como mudanças de hábitos e de estilos de vida.

Na população de hipertensos do estudo houve diferenças quanto às fontes de obtenção de medicamentos, em relação ao sexo: as mulheres obtiveram os medicamentos com maior frequência nas US, e os homens nas drogarias/farmácias e outras fontes. Segundo a literatura, as mulheres utilizam os serviços de saúde em maior proporção do que os homens 5, e estes priorizam as farmácias/drogarias e outros locais para obter medicamentos 19. Sawyer et al. 20 analisaram o perfil de utilização de serviços de saúde no Brasil e verificaram que, quando enfermos, os homens mais jovens procuram, inicialmente, farmácias e prontos-socorros.

Já 45,8\% dos hipertensos obtêm seus medicamentos nas US da rede pública; este percentual ascende a $54,4 \%$ entre os diabéticos. Tal resultado poderia sugerir, em alguma medida, maior efetividade em estratégias de implementação de programas voltados aos pacientes diabéticos, na atenção básica, no que se refere ao acesso aos medicamentos.

Tanto para hipertensos quanto para diabéticos que referiram cor da pele preta ou parda, as maiores prevalências de obtenção foram observadas nas US. Por outro lado, nas farmácias credenciadas ao PFPB e nas drogarias/farmácias não credenciadas e em outras fontes, as prevalências apresentaram-se significativamente menores em comparação aos brancos. A maior probabilidade de utilização de serviços públicos de saúde, por indivíduos pretos e pardos, constitui um indicativo do atendimento nas US de grupos com inserção socioeconômica historicamente menos favorecida no país.

Esse achado é condizente com a observação de redução da obtenção do medicamento nas US, com o aumento da escolaridade, e aumento significativo de obtenção nas drogarias/farmácias credenciadas ao PFPB e não credenciadas ao programa e outras fontes. A maioria da população atendida no serviço público de saúde é constituída de parcelas expressivas dos segmentos de menor renda, entre os quais a obtenção gratuita de medicamentos apresenta-se, frequentemen- 
te, como opção preferencial ou única de acesso aos tratamentos medicamentosos 21 .

Hipertensos e diabéticos sem plano de saúde obtiveram, em maior proporção $(66,4 \%$ e $75,1 \%$, respectivamente), seus medicamentos nas US, porém $23,9 \%$ dos hipertensos e $13,2 \%$ dos diabéticos sem plano de saúde recorreram às drogarias/farmácias e outras fontes. Quanto aos entrevistados filiados a plano de saúde, em maior proporção eles obtiveram seus medicamentos nas drogarias/farmácias não vinculadas ao PFPB e outros locais, no entanto, $20,8 \%$ dos hipertensos e $32,4 \%$ dos diabéticos com planos de saúde conseguiram seus medicamentos nas US. Também os percentuais de pacientes que obtiveram medicamentos nas farmácias/drogarias do PFPB foram mais elevados entre os que têm plano de saúde em comparação ao segmento que não têm.

Os dados deste estudo apontam que os segmentos socialmente mais vulneráveis (com menor escolaridade, cor da pele preta/parda e sem plano de saúde) obtêm, prioritariamente, seus medicamentos nas US. Assim, investimentos na qualificação da assistência farmacêutica prestada nessas unidades pode beneficiar em especial esses segmentos que são os socialmente mais vulneráveis e mais acometidos por doenças e mortes precoces, objetivando a redução das inequidades existentes.

No estudo, foram analisadas as fontes de obtenção de medicamentos segundo as regiões brasileiras. Para hipertensos, os maiores percentuais de obtenção de medicamentos em US foram identificados nas capitais das regiões Sul $(49,6 \%)$ e Sudeste $(48,8 \%)$, e para os diabéticos, os maiores percentuais ocorreram nas regiões Sul, Sudeste e Centro-oeste. Tal dado sugere que, em regiões social e economicamente mais desenvolvidas do país (Sudeste, Sul e Centro-oeste), a rede de atenção básica de saúde estaria mais organizada, de forma a proporcionar melhores condições de acesso ao medicamento a hipertensos e diabéticos.

Em todas as regiões, os percentuais de obtenção de medicamentos nas US, mais elevados para diabéticos em comparação com hipertensos, sugerem maior disponibilidade de medicamentos para o tratamento dos portadores de diabetes. Outras explicações decorrem de ofertas dos insumos para o controle do diabetes nas US, como as seringas com agulha acoplada para aplicação de insulina, tiras reagentes de medida de glicemia capilar e lancetas para punção digital, além de outros serviços assistenciais que resultam em maior vinculação do usuário às US.

As diferenças observadas entre as capitais podem refletir a cobertura e organização da aten- ção básica nestas cidades, assim como da assistência farmacêutica. As mesmas capitais tendem a apresentar menor percentual de obtenção de medicamentos nas US, seja para pacientes hipertensos ou para diabéticos, e nelas o maior percentual de pacientes é obrigado a arcar com os custos dos medicamentos. Constata-se a necessidade de investigações que avaliem os fatores e causas que possam explicar a heterogeneidade de acesso aos medicamentos para hipertensão e diabetes entre as capitais brasileiras.

Observa-se que, para ambas as doenças, os percentuais de obtenção, por meio de farmácias credenciadas ao PFPB, foram maiores em capitais com baixos percentuais de obtenção em US, como Natal, Rio de Janeiro, Palmas (Tocantins) e Belém. Tal ocorrência poderia explicar, parcialmente, a maior busca pelas farmácias do PFPB, uma vez que, desde 2011, estes estabelecimentos farmacêuticos proporcionam um conjunto de medicamentos para hipertensão e diabetes gratuitamente, viabilizando à população dessas capitais o acesso a medicamentos prescritos, sem desembolso de recursos próprios. Mas, nessas capitais, mais de $30 \%$ dos pacientes com diabetes e $50 \%$ daqueles com hipertensão precisam pagar pelo medicamento que utilizam para essas doenças.

A agilidade e praticidade em monitorar tendências das DCNT, na população adulta das 26 capitais brasileiras e do Distrito Federal, tornam o sistema VIGITEL um destacado instrumento de monitoramento de fatores de risco e proteção para doenças crônicas não transmissíveis no país 22 . Ainda que o alcance da telefonia fixa represente uma limitação, o uso de ponderações tende a reduzir os vícios resultantes da cobertura desigual em diferentes cidades. Em relação ao monitoramento de indivíduos em tratamento medicamentoso e das fontes de obtenção de medicamentos para hipertensão e diabetes, o sistema VIGITEL apresenta limitações que devem ser consideradas na análise realizada, pois não se investiga quantos e quais medicamentos são utilizados pelo paciente e se a fonte de obtenção difere entre eles. Porém, considerando o alto custo e a difícil operacionalização de inquéritos domiciliares específicos e aprofundados sobre acesso e uso de medicamentos, as informações desta pesquisa são essenciais para o planejamento, monitoramento e avaliação de algumas das ações propostas no âmbito da política de medicamentos do país 14 . Entre as limitações do presente estudo, deve ainda ser considerado que podem ter ocorrido erros de informações sobre a fonte de obtenção dos medicamentos. Existem farmácias comerciais denominadas "Farmácia Popular" que podem não 
ser credenciadas ao PFPB rede própria e não as farmácias credenciadas.

\section{Conclusão}

Este trabalho indicou disparidades nas fontes de obtenção de medicamentos para hipertensão e diabetes entre as capitais e regiões brasileiras e entre segmentos sociais da população, no período analisado, sendo que em capitais e regiões de menor desenvolvimento econômico, com maiores parcelas da população socialmente vulnerável, maior percentual de pacientes hipertensos e diabéticos precisou arcar com os custos dos medicamentos.

\section{Colaboradores}

K. S. Costa participou da concepção e delineamento do estudo, análise e interpretação dos dados, redação e revisão crítica do conteúdo intelectual do manuscrito e aprovação final da versão a ser publicada. P. M. S. B. Francisco colaborou na análise e interpretação dos dados e revisão do manuscrito. D. C. Malta e M. B. A. Barros participaram da concepção e delineamento do estudo, análise e interpretação dos dados e aprovação final da versão a ser publicada.
A análise dos padrões de acesso a medicamentos anti-hipertensivos e antidiabéticos identificados neste estudo pode propiciar subsídios para o redirecionamento das políticas públicas, no sentido de favorecer a promoção de ações de saúde e de assistência farmacêutica mais equânimes no país. Os resultados sinalizam também a necessidade do desenvolvimento de pesquisas sobre a organização dos serviços de assistência farmacêutica no país, incluindo as farmácias do PFPB, visando à melhor compreensão a respeito das barreiras ao acesso e das disparidades existentes.

\section{Agradecimentos}

As autoras do artigo agradecem às equipes técnicas da Secretaria de Vigilância em Saúde do Ministério de Saúde, envolvidas na implantação e operacionalização do sistema VIGITEL. 


\section{Referências}

1. World Health Organization. Non communicable diseases country profiles 2011. Geneva: World Health Organization; 2011.

2. Goulart FAA. Doenças crônicas não transmissíveis: estratégias de controle e desafios para o sistema de saúde. Brasília: Organização Pan-Americana da Saúde/Ministério da Saúde; 2011.

3. Departamento de Análise de Situação de Saúde, Secretaria de Vigilância em Saúde, Ministério da Saúde. Plano de Ações Estratégicas para Enfrentamento das Doenças Crônicas Não Transmissíveis (DCNT) no Brasil 2011-2022. Brasília: Ministério da Saúde; 2011.

4. Barros MBA, Francisco PMSB, Zanchetta LM, César CLG. Tendências das desigualdades sociais e demográficas na prevalência de doenças crônicas no Brasil, PNAD: 2003-2008. Ciênc Saúde Coletiva 2011; 16:3755-68.

5. Gontijo MF, Ribeiro AQ, Klein CH, Rozenfeld S, Acurcio FA. Uso de anti-hipertensivos e antidiabéticos por idosos: inquérito em Belo Horizonte, Minas Gerais, Brasil. Cad Saúde Pública 2012; 28:1337-46.

6. Malta DC, Silva Junior JB. Brazilian Strategic Action Plan to combat Chronic Non-Communicable Diseases and the global targets set to confront these diseases by 2025: a review. Epidemiol Serv Saúde 2013; 22:151-64.

7. Ministério da Saúde. Política Nacional de Medicamentos. Brasília: Ministério da Saúde; 2001. (Série C. Projetos, Programas e Relatórios, 25).

8. Conselho Nacional de Saúde. Resolução no 338, de 2004, de 06 de maio de 2004. Aprova a Política Nacional de Assistência Farmacêutica e estabelece seus princípios gerais e eixos estratégicos. Diário Oficial da União 2004; 20 mai.

9. Ministério da Saúde. Portaria no 1.214, de 13 de junho de 2012. Institui o Programa Nacional de Qualificação da Assistência Farmacêutica no âmbito do SUS. Diário Oficial da União 2012; 14 jun.

10. Ministério da Saúde. Decreto no 5.090, de 20 de maio de 2004. Regulamenta a Lei no 10.858 , de 13 de abril de 2004, e institui o programa "Farmácia Popular do Brasil”, e dá outras providências. Diário Oficial da União 2004; 21 mai.

11. Ministério da Saúde. Portaria no 491, de 9 de março de 2006. Dispõe sobre a expansão do Programa "Farmácia Popular do Brasil". Diário Oficial da União 2006; 10 mar.

12. Ministério da Saúde. Pesquisa Nacional sobre Acesso, Utilização e Promoção do Uso Racional de Medicamentos: projeto de pesquisa. Brasília: Ministério da Saúde; 2013.
13. Luiza VL, Bermudez JAZ. Acesso a medicamentos: conceitos e polêmicas. In: Bermudez JAZ, Oliveira MA, Escher A, organizadores. Acceso a medicamentos: derecho fundamental, papel del Estado. Rio de Janeiro: Fundação Oswaldo Cruz/Brasília: Organização Pan-Americana da Saúde/Organização Mundial da Saúde; 2004. p. 45-67.

14. Secretaria de Gestão Estratégica e Participativa, Secretaria de Vigilância em Saúde, Ministério da Saúde. VIGITEL Brasil 2011: Vigilância de Fatores de Risco e Proteção para Doenças Crônicas por Inquérito Telefônico. Brasília: Ministério da Saúde; 2012.

15. Barros AJ, Hirakata VN. Alternatives for logistic regression in cross-sectional studies: an empirica comparison of models that directly estimate the prevalence ratio. BMC Med Res Methodol 2003; 3:21.

16. Duncan BB, Chor D, Aquino EML, Bensenor IM, Mill JG, Schmidt MI, et al. Doenças crônicas não transmissíveis no Brasil: prioridade para enfrentamento e investigação. Rev Saúde Pública 2012; 46 Suppl 1:126-34.

17. Ben AJ, Neumann CR, Mengue SS. Teste de Morisky-Green e Brief Medication Questionnaire para avaliar adesão a medicamentos. Rev Saúde Pública 2012; 46:279-89.

18. Wang YR, Alexander GC, Stafford RS. Outpatient hypertension treatment, treatment intensification, and control in Western Europe and the United States. Arch Intern Med 2007; 167:141-7.

19. Vosgerau MZS, Soares DA, Souza RKT, Matsuo T, Carvalho GS. Consumo de medicamentos entre adultos na área de abrangência de uma Unidade de Saúde da Família. Ciênc Saúde Coletiva 2011; 16 Suppl 1:1629-38.

20. Sawyer DO, Leite IC, Alexandrino R. Perfis de utilização dos serviços de saúde no Brasil. Ciênc Saúde Coletiva 2002; 7:757-76.

21. Paniz VMV, Fassa AG, Facchini LA, Bertoldi AD, Piccini RX, Tomasi E, et al. Acesso a medicamentos de uso contínuo em adultos e idosos nas regiões Sul e Nordeste do Brasil. Cad Saúde Pública 2008; 24:267-80.

22. Moura EC, Silva SA, Malta DC, Morais Neto OL Fatores de risco e proteção para doenças crônicas: vigilância por meio de inquérito telefônico, VIGITEL, Brasil, 2007. Cad Saúde Pública 2011; 27: 486-96. 


\section{Abstract}

This study aimed to analyze differences between patients with diabetes and hypertension in drug treatment and their sources for obtaining medication. This was a cross-sectional study with data from the VIGITEL telephone survey in 2011 in Brazil's state capitals and Federal District. Some 72\% of the 15,027 hypertensive patients and $78.2 \%$ of the 4,083 diabetics were on medication; $45.8 \%$ of the hypertensive patients obtained their medications from public health units, 15.9\% from the Popular Pharmacy program, and $38.3 \%$ from drugstores, pharmacies, and other sources. The rates among diabetics were $54.4 \%, 16.2 \%$, and $29.4 \%$, respectively. In the public health units the percentages were highest among individuals with less schooling, black or brown skin, and without private health plans, while the percentages in the Popular Pharmacy program and drugstores/pharmacies and other sources were higher among individuals with more schooling, white skin, and private health plans. Access to different sources of medicines showed disparities between Brazil's regions and state capitals and between social segments of the population.

Drug Utilization; Health Services Accessibility; Hypertension; Diabetes Mellitus

\section{Resumen}

Analizar las diferencias entre los diabéticos e hipertensos, en relación con el tratamiento farmacológico y sus fuentes de obtención. Se trata de un estudio transversal con datos de VIGITEL del 2011 en capitales brasileñas. Cerca del 72\% de los 15.027 hipertensos y el $78,2 \%$ de los 4.083 diabéticos estaban en tratamiento; un 45,8\% de los pacientes hipertensos contaba con la medicación en las unidades de salud; 15,9\% en farmacias populares; $y$ un 38,3\% en farmacias y otras fuentes y entre los diabéticos, $54,4 \% ; 16,2 \%$; y 29,4\%, respectivamente. En las unidades de salud los porcentajes fueron más altos entre los menos educados, color de piel negro/mulato y sin plan de salud, mientras que la obtención en Farmacia Popular y otras fuentes fueron más altas entre los más educados, de piel blanca y con el plan privado. El acceso a diferentes fuentes de obtención mostró diferencias entre las regiones y las capitales brasileñas y segmentos sociales de la población.

Utilización de Medicamentos; Accesibilidad a los Servicios de Salud; Hipertensión; Diabetes Mellitus

Recebido em 15/Jun/2014

Versão final reapresentada em 07/Jun/2015

Aprovado em 17/Jun/2015 